EPJ Web of Conferences 78, 03003 (2014)

DOI: $10.1051 /$ epjconf / 20147803003

(C) Owned by the authors, published by EDP Sciences, 2014

\title{
Poisson Geometry of Difference Lax Operators, and Difference Galois Theory, or Quantum groups from Poisson brackets anomalies
}

\author{
Michael Semenov-Tian-Shansky \\ Institut Mathématique de Bourgogne, Dijon, France \\ and St.Petersburg Department of Steklov Mathematical Institute \\ St.Petersburg, Russia
}

\begin{abstract}
We discuss the lift of Poisson structures associated with auxiliary linear problems for the differential and difference Lax equations to the space of wave functions. Due to a peculiar symmetry breaking, the corresponding differential and difference Galois groups become Poisson Lie Groups.
\end{abstract}

\section{Introduction}

It is well known that the transition from Classical to Quantum Mechanics is best described in terms of the deformation of the associated algebras of observables. In a wide class of examples Quantization does not affect the symmetry: both classical and quantum mechanical systems share the same symmetry groups. Since the discovery of Quantum Groups in early 1980's we know that this need not always be the case: there exist natural quantum deformations of Lie groups and, in particular, of those which arise as symmetry groups of fundamental classical and quantum mechanical systems. So why are symmetry groups not deformed when we pass from Classical to Quantum Mechanics? The answer is of course very simple. Quantization of Lie groups fits into the general paradigm of Deformation Quantization: its semiclassical counterpart is Poisson structure on the underlying group manifolds which equips them with the structure of Poisson Lie groups. Natural action of symmetry groups on the classical phase spaces preserve the Poisson brackets, and hence the Poisson structure on the symmetry group itself is identically zero. As a result, quantum deformation which transforms classical observables into quantum ones does not affect the symmetry group. However, there exist non-trivial examples when a natural group action does not preserve the Poisson brackets. An interesting example of such Poisson brackets anomaly arises in the theory of the celebrated Korteweg-de Vries equation; here the dynamical symmetry group which becomes a Poisson group (and hence eventually a quantum group) is the differential Galois group associated with the auxiliary linear problem.

Recall that the phase space of the KdV equation $u_{t}=6 u u_{x}+u_{x x x}$ may be identified with the space of Schroedinger operators with potential $u$ on the line or on the circle. The algebra of observables consists of local functionals of $u$. A natural Poisson structure for the potentials is related to the famous Virasoro algebra (for this reason the $\mathrm{KdV}$ phase space is also related 
to models of $2 \mathrm{~d}$-gravity). It is natural to ask whether there is a natural Poisson structure on the space of wave functions of a Schroedinger operator. This question is in fact of practical interest in application to a family of $\mathrm{KdV}$-like equation.

Let us recall a nice geometric way to restore the potential from the wave functions [7]. For a given $u$ the space of solutions of the Schroedinger equation $-\psi^{\prime \prime}-u \psi=0$ is 2-dimensional; any two solutions $\phi, \psi$ have constant wronskian $W=\phi \psi^{\prime}-\phi^{\prime} \psi$. Set $\eta(x)=\phi(x) / \psi(x)$. The potential $u$ may be restored from $\eta$ by the formula $u=\frac{1}{2} S(\eta)$, where

$$
S(\eta)=\frac{\eta^{\prime \prime \prime}}{\eta^{\prime}}-\frac{3}{2}\left(\frac{\eta^{\prime \prime}}{\eta^{\prime}}\right)^{2}
$$

is the Schwarzian derivative. A change of basis amounts to linear transformation $(\phi, \psi) \mapsto$ $(\phi, \psi) g, g \in S L(2)$ and to fractional linear transformation of $\eta=\phi / \psi . S(u)$ is projective invariant: if $\tilde{\eta}=\frac{a \eta+c}{b \eta+d}$, then $S(\tilde{\eta})=S(u)$. The $\mathrm{KdV}$ equation is included into an interesting family of "KdV-like equations" related to projective group and its subgroups. Atop the tower of these "KdV-like equations" is the "Schwarz-KdV equation" $\eta_{t}=S(\eta) \eta_{x}$. The projective group $P S L(2)$ is acting on its solutions: if $\eta$ is its solution, so is $\frac{a \eta+c}{b \eta+d}$ for all $g=\left(\begin{array}{ll}a & b \\ c & d\end{array}\right) \in S L(2)$. Original KdV holds for $u=S(\eta)$ which is $P S L(2)$-invariant. Other KdV-like equations hold for the invariants of various subgroups of $P S L(2)$.

To put it more formally, we identify observables of $\mathrm{KdV}$ with local densities which are rational functions of $u$ and its derivatives; in a similar way, observables for Schwarz-KdV are rational functions of $\eta$ and its derivatives. The group $G=P S L(2)$ acts on this algebra of observables and commutes with derivation $\partial_{x}$. We define the differential field $\mathbb{C}\left\langle\psi_{1}, \psi_{2}\right\rangle$ as a free algebra of rational functions in an infinite set of variables $\psi_{1}, \psi_{2}, \psi_{1}^{\prime}, \psi_{2}^{\prime}, \psi_{1}^{\prime \prime}, \psi_{2}^{\prime \prime}, \ldots$ with a formal derivation $\partial$ such that $\partial \psi_{i}^{(n)}=\psi_{i}^{(n+1)}$. A differential automorphism is an automorphism of $\mathbb{C}\left\langle\psi_{1}, \psi_{2}\right\rangle$ (as an algebra) which commutes with $\partial$. All differential automorphisms are induced by linear transformations $\left(\psi_{1}, \psi_{2}\right) \mapsto\left(\psi_{1}, \psi_{2}\right) \cdot g, g \in G L(2, \mathbb{C})$. Let $(W)$ be the differential ideal in $\mathbb{C}\left\langle\psi_{1}, \psi_{2}\right\rangle$ generated by $\psi_{1} \psi_{2}^{\prime}-\psi_{1}^{\prime} \psi_{2}-1$. Automorphisms which preserve $W$ belong to $G=S L(2)$. The differential subfield of $G$-invariants coincides with $\mathbb{C}\langle u\rangle$. Let $Z=\{ \pm 1\}$ be the center of $G$ and $N, A, B=A N$ its standard subgroups (nilpotent, split Cartan \& Borel). The subfields of invariants are freely generated differential algebras: $\mathcal{C}\langle\phi, \psi\rangle^{Z}=\mathcal{C}\langle\eta\rangle, \quad \eta=\phi / \psi, \mathcal{C}\langle\eta\rangle^{A}=\mathcal{C}\langle\rho\rangle, \quad \rho=\eta^{\prime} / \eta, \mathcal{C}\langle\eta\rangle^{N}=\mathcal{C}\langle\theta\rangle, \quad \theta=\eta^{\prime}$, $\mathcal{C}\langle\eta\rangle^{B}=\mathcal{C}\langle v\rangle, \quad v=\eta^{\prime \prime} / \eta^{\prime}, \mathcal{C}\langle\eta\rangle^{G}=\mathcal{C}\langle u\rangle, \quad u=S(\eta)$. The tower of compatible integrable $\mathrm{KdV}$-like equations associated with subgroups of $P S L(2)$ is given in the following diagram:

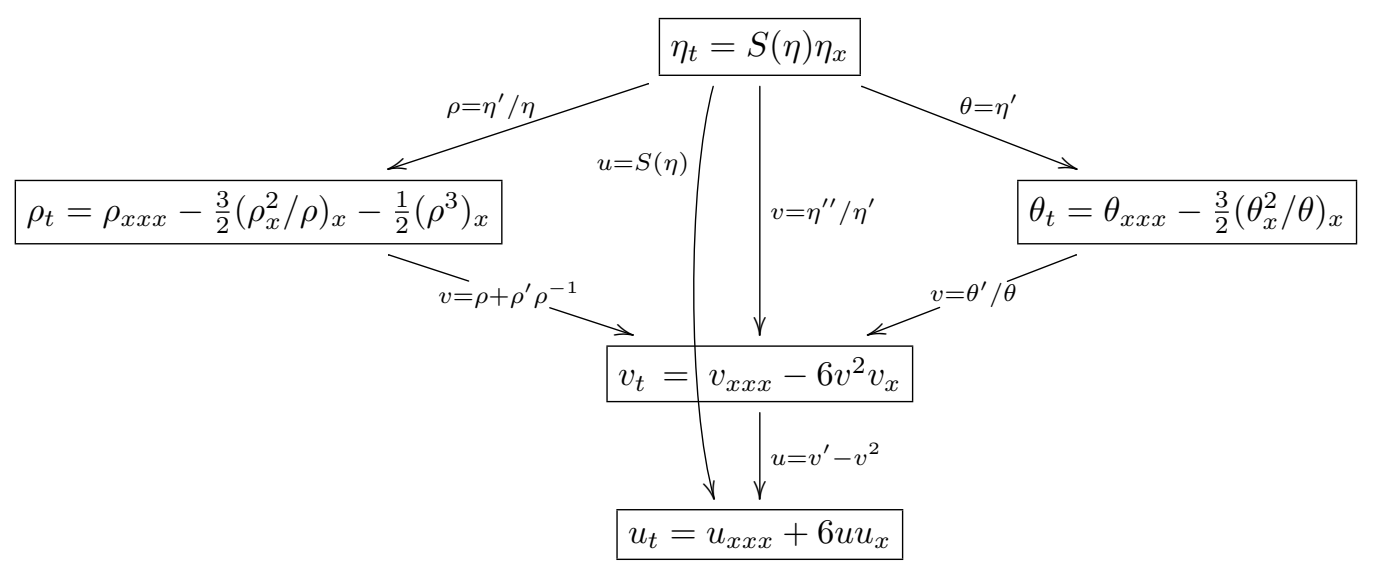


The corresponding tower of differential extensions is described by the commutative diagram

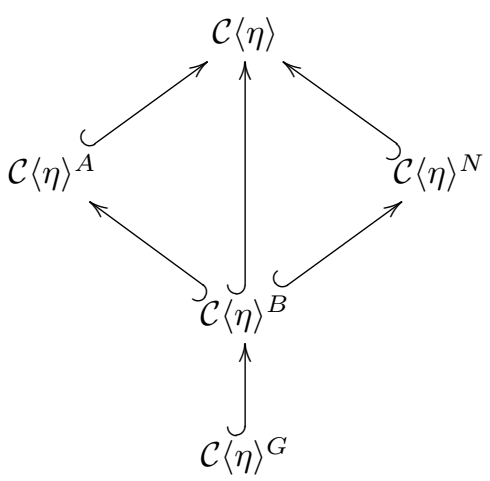

Inclusions in this diagram match with "differential substitutions" listed above and with the tower of $\mathrm{KdV}$-like equations

The natural question is to put this picture (first suggested by G.Wilson [9]) into the Hamiltonian framework.

As well known, the space of second order differential operators carries a natural Poisson structure (incidentally, this is the Poisson-Virasoro algebra).

Question: Extend this Poisson structure to the space of wave functions in such a way that all arrows in the diagram above become Poisson maps.

The answer to this question [6] is non-trivial, as all arrows in the diagram above go in wrong direction (Poisson structure cannot be pulled back!). Still, the lift is possible (and even almost unique); however, the resulting brackets are anomalous: they are not projective invariant. Instead, a new axiom holds true: the projective group becomes a Poisson Lie group (and eventually a quantum group); all brackets are Poisson covariant.

$$
\{\eta(x), \eta(y)\}=\eta(x)^{2}-\eta(y)^{2}-\operatorname{sign}(x-y)(\eta(x)-\eta(y))^{2} .
$$

For $\theta=\eta^{\prime}$ we have

$$
\{\theta(x), \theta(y)\}=2 \operatorname{sign}(x-y) \theta(x) \theta(y) .
$$

For $v=\frac{1}{2} \eta^{\prime \prime} / \eta^{\prime}=\frac{1}{2} \theta^{\prime} / \theta$ we have

$$
\{v(x), v(y)\}=\frac{1}{2} \delta^{\prime}(x-y) .
$$

For $u=\frac{1}{2} v^{\prime}-v^{2}=S(\eta)$ we have

$$
\{u(x), u(y)\}=\frac{1}{2} \delta^{\prime \prime \prime}(x-y)+\delta^{\prime}(x-y)[u(x)+u(y)] .
$$

In these formulas $\operatorname{sign}(x-y)$ is the distribution kernel of the operator $\partial_{x}^{-1}$ and $\delta^{\prime}(x-y)$ is the distribution kernel of $\partial_{x}$. The Poisson bracket for $u$ is the Poisson-Virasoro algebra.

Poisson brackets for $\eta$ are not projective invariant: there are correction terms which mean that this action is Poisson, with the group $S L(2)$ itself carrying the so called standard (Sklyanin) Poisson bracket [8].

In a sense, one has to guess the Poisson brackets for $\eta$. The situation becomes more transparent if we pass to first order matrix differential operators and then to difference operators. As well known, second order differential operators may be written as first order $2 \times 2$ matrix differential operators. One can start with arbitrary first order matrix differential operators; 
to get the correct Poisson bracket relations for second order operators one needs a reduction procedure (Drinfeld-Sokolov theory [3]). Again, the choice of a Poisson structure in the space of wave functions is nontrivial. The Poisson structure associated with first order matrix difference operators reveals similar anomalies which are the main subject of the present talk.

\section{Abstract difference operators}

To set up a general framework for the difference case let us assume that $\mathbb{G}$ is a Lie group equipped with an automorphism $\tau$. Let $G=\mathbb{G}^{\tau}$ be the group of "quasi-constants", $G=$ $\left\{g \in \mathbb{G} ; g^{\tau}=g\right\}$. The "auxiliary linear problem" reads:

$$
\psi^{\tau} \psi^{-1}=L
$$

There is a natural action of $\mathbb{G}$ on itself by left multiplication which induces gauge transformations for $L$ :

$$
g: \psi \mapsto g \cdot \psi, L \mapsto g^{\tau} L g^{-1} .
$$

The quasi-constants act by right multiplications, $\psi \mapsto \psi h$ and leave $L$ invariant.

There are several natural realizations of this scheme:

- $\mathbb{G}$ consists of functions on a lattice $\mathbb{Z}$ with values in a matrix group $G$ and $\tau$ is a shift operator; it is also possible to introduce multi-dimensional lattices with several commuting shift automorphisms.

- $\mathbb{G}$ consists of functions on the line with $g^{\tau}(x)=g(x+1)$.

- $\mathbb{G}$ consists of functions which are meromorphic in $\mathbb{C}^{*}$ and $\tau$ acts by $g^{\tau}(z)=g(q z), q \neq 1$.

In the first case, the group of quasi-constants consists of genuine constant functions on the lattice with values in $G$, in the second case it consists of $G$-valued periodic functions, in the 3 d case it consists of $G$-valued functions on the elliptic curve $E_{q}=\mathbb{C}^{*} / q^{\mathbb{Z}}$.

Recall that the space of 1st order differential operators on the line carries a natural Poisson structure (Schwinger bracket) and the gauge action is Hamiltonian. In the difference case the gauge action is not Hamiltonian; rather, the gauge group is a Poisson Lie group with a Poisson structure determined by the choice of a classical r-matrix. Once the Poisson group structure on the gauge group is chosen, there is a unique Poisson structure on the space of 1st order difference operators which is Poisson covariant with respect to the gauge action. This is the "discrete current algebra" first described in [1] (both in Poisson and in Quantum group setting). This Poisson structure may be lifted to the space of wave functions; its choice is guided by the two natural requirements: it should be Poisson covariant with respect to left translations (gauge action) and right translations and yield the correct Poisson structure on the space of potentials $L=\psi^{\tau} \psi^{-1}$.

Definition. For $f \in \operatorname{Fun}(\mathbb{G})$ we denote by $\nabla_{f}, \nabla_{f}^{\prime}$ its left and right gradients defined by

We put

$$
\left\langle\nabla_{f}(\psi), \xi\right\rangle=\left.\frac{d}{d t}\right|_{t=0} f\left(e^{t \xi} \psi\right), \quad\left\langle\nabla_{f}^{\prime}(\psi), \xi\right\rangle=\left.\frac{d}{d t}\right|_{t=0} f\left(\psi e^{t \xi}\right) .
$$

$$
\left\{f_{1}, f_{2}\right\}=\left\langle l\left(\nabla_{f_{1}}\right), \nabla_{f_{2}}\right\rangle+\left\langle r\left(\nabla_{f_{1}}^{\prime}\right), \nabla_{f_{2}}^{\prime}\right\rangle .
$$

Here $l$ and $r$ are two (a priori, different) classical $\mathrm{r}$-matrices. This may be regarded as an abstract version of the "exchange algebra" introduced in the early 1990's [2]. The main 
question, of course, is to restrict and explain the choice of $l$ and $r$. For a given $l$ the gauge action becomes Poisson if the gauge group carries the Sklyanin bracket associated with $l$. Left and right bracket are almost independent, but they are linked via the Jacoby/Yang-Baxter identity.

A simple computation. We look at the contribution of $l$ to the Poisson brackets of potentials. Suppose that $f(\psi)=F\left(\psi^{\tau} \psi^{-1}\right)$. We denote by $X_{F}, X_{F}^{\prime}$ left and right gradients of $F$. Then

$$
\left\{f_{1}, f_{2}\right\}^{l}(\psi)=\left\langle l\left(X_{1}\right), X_{2}\right\rangle+\left\langle l\left(X_{1}^{\prime}\right), X_{2}^{\prime}\right\rangle-\left\langle l \circ \tau^{-1}\left(X_{1}\right), X_{2}^{\prime}\right\rangle-\left\langle\tau \circ l\left(X_{1}^{\prime}\right), X_{2}\right\rangle .
$$

This formula resembles the "discrete current algebra" referred to above [1], but it lacks some crucial terms which arise from the contribution of $r$. The choice of $r$ appears to be very rigid.

Indeed, left gradient of $f(\psi)=F\left(\psi^{\tau} \psi^{-1}\right)$ depends only on left and right gradients of $F$ (regarded as a function of $L=\psi^{\tau} \psi^{-1}$ ). By contrast, its right gradient depends on $\psi$. After some calculations, one gets explicitly:

$$
\left\{f_{1}, f_{2}\right\}^{r}(\psi)=\left\langle\left(r-\tau \cdot r+r-r \cdot \tau^{-1}\right) \operatorname{Ad} \psi^{-1} X_{1}^{\prime}, \operatorname{Ad} \psi^{-1} X_{2}^{\prime}\right\rangle .
$$

Our mapping is Poisson if and only if $\operatorname{Ad} \psi^{-1}$ cancels. Remarkably, this cancellation is achieved by a simple and unique choice of $r$.

Proposition. Let

$$
r=(\tau+I)(\tau-I)^{-1}
$$

be a partially defined linear operator with $\operatorname{Dom} r=\operatorname{Im}(\tau-I)$. It is skew iff $\tau$ is orthogonal and satisfies the modified classical Yang-Baxter identity

$$
[r X, r Y]-r([r X, Y]+[X, r Y])+[X, Y]=0
$$

With our choice of $r$, the contribution of the right bracket finally becomes

$$
\left\{f_{1}, f_{2}\right\}^{r}(\psi)=\left\langle X_{1}, \tau X_{2}^{\prime}\right\rangle-\left\langle\tau X_{1}^{\prime}, X_{2}\right\rangle .
$$

The above formula precisely provides the missing terms to convert the left bracket for $L$ 's into the correct lattice algebra. If $l$ also satisfies the modified Yang-Baxter identity, the full bracket satisfies Jacoby identity.

Formula (2) defines $r$ as a singular integral operator; its regularization amounts to a correct treatment of the subspace of quasi-constants.

Example: $\tau$ is a shift operator on the line, $\tau f(x)=f(x+1)$. Then formally

$$
r f(x)=\frac{1}{2 \pi} \int_{-\infty}^{\infty} \operatorname{cotan}(k / 2) \hat{f}(k) e^{i k x} d k,
$$

where $\hat{f}$ is the Fourier transform of $f$. Let us choose a finite dimensional r-matrix $r_{0}$ acting in the space of values. Put

$$
F(x)=\sum_{n=-\infty}^{\infty} f(x+n)=\sum_{n=-\infty}^{\infty} \hat{f}(2 \pi n) e^{2 \pi i n x}
$$

$F$ is a quasi-constant lying in the kernel of $\tau$. The regularization of $r$ is given by

$$
(r f)(x)=r_{0}(F(x))+\text { v.p. } \frac{1}{2 \pi} \int_{-\infty}^{\infty} \operatorname{cotan}(k / 2) \hat{f}(k) e^{i k x} d k,
$$


where $r_{0}$ is acting pointwise in the subspace of quasi-constants. For q-difference operators the kernel of $r$ is expressed through theta functions. In both difference and q-difference cases the resulting bracket is Poisson covariant with respect to the right action of group of constant matrices; generically, it is isomorphic to the difference Galois group [4].

In the general case, the choice of $l$ and $r_{0}$ remains arbitrary. In the important case of higher order scalar difference operators (difference Drinfeld-Sokolov theory [5]) this choice is further restricted by a peculiar Poisson anomaly which allows to fix both $l$ and $r_{0}$ completely. The Poisson structure in the space of second order difference operators is the deformed Poisson Virasoro algebra; its extension to the space of projective wave functions is again possible and has the expected Poisson covariance properties with respect to the action of the difference Galois group. The explicit formulae will be presented in a separate publication.

\section{References}

[1] Alekseev, A., Faddeev, L., Semenov-Tian-Shansky, M., Hidden quantum group inside Kac-Moody algebras, Commun. Math. Phys. 149 (1992), 335-345.

[2] Babelon, O. Exchange formula and lattice deformation of the Virasoro algebra, Phys. Lett. B 238 (1990), no. 2-4, 234-238.

[3] Drinfeld, V.G., Sokolov, V.V. Lie algebras and equations of Korteweg-de Vries type, Sov. Math. Dokl. 23 (1981) 457-462.

[4] Etingof P. Galois groups and connections matrices of q-difference equations, Electronic Res. Announc. of the Amer. Math. Soc. 1, 1 (1995).

[5] Frenkel, E., Reshetikhin, N., Semenov-Tian-Shansky, M.A. Drinfeld-Sokolov reduction for difference operators and deformations of $W$-algebras. I: The case of Virasoro algebra, Commun. Math. Phys. 192, (1998) 605-629.

[6] Marshall I., Semenov-Tian-Shansky, M.A. Poisson groups and differential Galois theory of Schrödinger equation on the circle, Commun. Math. Phys. 2008, 284, 537552.

[7] Ovsienko, V., Tabachnikov, S. Projective differential geometry old and new: from Schwarzian derivative to cohomology of diffeomorphism group. Cambridge Tracts in Mathematics, 165. Cambridge University Press, Cambridge, 2005.

[8] Semenov-Tian-Shansky, M.A. Dressing transformations and Poisson-Lie group actions, Publ. RIMS. 21 (1985), 1237-1260.

[9] Wilson, G. On the quasi-Hamiltonian formalism of the KdV equation, Physics Letters A 132 (1988), 445-450. 